\title{
ON MEASURE REPLETENESS AND SUPPORT FOR LATTICE REGULAR MEASURES
}

\author{
GEORGE BACHMAN \\ Department of Mathematics \\ Polytechnic Institute of New York \\ Brooklyn, NY 11201 U.S.A. \\ P.D. STRATIGOS \\ Department of Mathematics \\ Long Island University \\ Brooklyn, NY 11201 \\ (Received October 31, 1984)
}

\begin{abstract}
The present paper is mainly concerned with establishing conditions which assure that all lattice regular measures have additional smoothness properties or that simply all two-valued such measures have such properties and are therefore Dirac measures. These conditions are expressed in terms of the general Wallman space. The general results are then applied to specific topological lattices, yielding new conditions for measure compactness, Borel measure compactness, clopen measure repleteness, strong measure compactness, etc. In addition, smoothness properties in the general setting for lattice regular measures are related to the notion of support, and numerous applications are given.
\end{abstract}

KEY WORDS AND PHRASES. Support of a measure, repleteness, realcompactness, and $\alpha-$ completeness, measure completeness, measure compactness, and Borel measure compactness, clopen measure repleteness, strong measure repleteness, strong measure compactness, etc. 1980 AMS SUBJECT CLASSIFICATION CODES. 28A60, 28 A32.

1. INTRODUCTION.

In an earlier paper [5], we obtained conditions for $\sigma$-smoothness, $\tau$-smoothness, and tightness of lattice regular measures. This was done in a general framework for a set $X$ and a lattice of subsets of $X, L$, which was just disjunctive and at times separating. The general approach was adopted so as to fit many topological lattices which are not $\delta$ or not normal. This approach was made possible by utilizing general lattice regular measure extension theorems (see [4]). Thus it was possible to bypass the general Alexandroff Representation Theorem [2] in which a delta normal lattice is needed. The results were then expressed in terms of $\operatorname{IR}(L)-X$, where $\operatorname{IR}(L)$ is the general Wallman space associated with the set $X$ and the lattice $L$. These results generalized known results pertaining to Baire measures and $B X-X$, where $B X$ is the Stone-Čech compactification of the Tychonoff space $X$. In particular, our general approach lead to new results pertaining to smoothness and tightness 
of closed regular Borel measures in just $T_{1}$ topolugical spaces expressible in terms of $\omega X-X$, where $\omega X$ is the Wallman compactification of $X$ and also to clopen regular Borel measures in o-dimensional $\mathrm{T}_{1}$ spaces expressible in terms of $\beta_{0} \mathrm{X}-\mathrm{X}$, where $\beta_{0} \mathrm{X}$ is the Banaschewski compactification of $\mathrm{X}$.

In the first part of this paper we utilize the framework of the previously mentioned paper and obtain new results for lattice repleteness, measure repleteness and strongly measure repleteness. We then apply these results to specific topological lattices and obtain new conditions for measure compactness, Borel measure compactness, and clopen measure repleteness and similar facts for strongly measure compactness, strongly Borel measure compactness, and strongly clopen measure repleteness. (See, in particular, Theorems 2.4, 2.6 and their consequences and associated examples.)

It is advantageous to be able to characterize various repleteness properties in terms of support of certain measures. We pursue this in general in the second part of the paper. We cite here just one of the more important results (see Theorem 3.3): If $L$ is separating and disjunctive, then $L$ is measure replete iff the support of every o-smooth, L-regular measure (which is not the zero measure) is nonempty. This result has many applications. Thus, in this part of the paper, we concentrate on various aspects of support of a measure.

2. TERMINOLOGY AND NOTATION.

I. Most of the terminology used in the present paper goes back to Wallman [10] and Alexandroff [1], [2]. Some of the more recent terminology appears in Noebeling [7] and Frolik [6], as well as in [5], [8]. For the reader's convenience, in this part we will collect some of the special terminology which is used throughout the paper.

Consider any set $X$ and any lattice of subsets of $X, L$. The algebra of subsets of $X$ generated by $L$ is denoted by $A(L)$. The o-algebra of subsets of $X$ generated by $L$ is denoted by $\sigma(L)$. Next, consider any algebra of subsets of $X, A$. A measure on $A$ is defined to be a function, $\mu$, from $A$ to $R$, such that $\mu$ is bounded and finitely additive. The set whose general element is a measure on $A(L)$ is denoted by $M(L)$. For the general element of $M(L), \mu$, the support of $\mu$ is defined to be $n\{L \in L /|\mu|(L)=|\mu|(X)\}$ and is denoted by $S(\mu)$. An element of $M(L)$, $\mu$, is said to be $L$-regular iff for every element of $A(L), E$, for every positive number, $\varepsilon$, there exists an element of $L, L$, such that $L \subset E$ and $|\mu(E)-\mu(L)|<\varepsilon$. The set whose general element is an element of $M(L)$ which is $L$-regular is denoted by $\operatorname{MR}(L)$. An element of $M(L), \mu$, is said to be $L-(\sigma-s m o o t h)$ iff for every sequence in $A(L),\left\langle A_{n}\right\rangle$, if $\left\langle A_{n}\right\rangle$ is decreasing and $\lim A_{n}=\emptyset$, then $\lim \mu\left(A_{n}\right)=0$. The set whose general element is an element of $M(L)$ which is $L-(\sigma$-smooth) is denoted by $M(\sigma, L)$. The set whose general element is an element of $M(L)$ which is $\sigma$-smooth just for $\left\langle A_{n}>\right.$ in $L$ is denoted by $M\left(\sigma^{*}, L\right)$. An element of $M(L), \mu$, is said to be $L-(\tau-s m o o t h)$ iff for every net in $L,\left\langle L_{\alpha}>\right.$, if $\left\langle L_{\alpha}>\right.$ is decreasing and 1 im $L_{\alpha}=\emptyset$, then $\lim \mu\left(\mathrm{L}_{\alpha}\right)=0$. The set whose general element is an element of $M(L)$ which is $L-(\tau-s m o o t h)$ is denoted by $M(\tau, L)$. An element of $M(L), \mu$, is said to be $L$-tight iff $\mu \in M(\sigma, L)$ and for every positive number, $\varepsilon$, there exists an $L$-compact set, $K$, such that $|\mu|_{*}(K)<\varepsilon$. The set whose general element is an ele- 
ment of $M(L)$ which is $L$-tight is denoted by $M(t, L)$. The set whose general element is an element of $M(L), \mu$, such that $\mu(A(L))=\{0,1\}$, that is, the set of $n-1$ measures is denoted by $I(L)$.

$L$ is said to be replete iff whenever an element of $I(L), \mu$, belongs to $\operatorname{IR}(\sigma, L)$, then $S(\mu) \neq \emptyset . L$ is said to be prime complete iff whenever an element of $I(L), \mu$, belongs to $I\left(\sigma^{*}, L\right)$, then $S(\mu) \neq \emptyset . L$ is said to be measure replete iff $\operatorname{MR}(\sigma, L)=\operatorname{MR}(\tau, L) . \quad L$ is said to be strongly measure replete iff $\operatorname{MR}(\sigma, L)=\operatorname{MR}(t, L)$. Next, consider any topological space $X$ and denote its collection of closed sets by $F$, its collection of open sets by 0 , its collection of clopen sets by $C$, and its collection of zero sets by $Z$. In case $X$ is $T_{3 \frac{1}{2}}$, $X$ is said to be realcompact iff $Z$ is replete. $X$ is said to be $\alpha$-complete iff $F$ is replete. $X$ is said to be $N-$ compact iff $C$ is replete. Moreover, $X$ is said to be measure compact iff $Z$ is measure replete. $X$ is said to be Borel measure compact iff $F$ is measure replete. $\mathrm{X}$ is said to be clopen measure replete iff $\mathrm{C}$ is measure replete.

NOTE. Since every element of $M(L)$ is expressible as the difference of nonnegative elements of $M(L)$, without loss of generality, we shall work with nonnegative elements of $M(L)$.

II. Among the principal tools utilized in the present work are three measures induced by the general element of $M(L)$, denoted by $\mu$; (these measures are denoted by $\hat{\mu}, \tilde{\mu}$, and $\left.\mu^{\prime}\right)$ and certain criteria for $\sigma$-smoothness, $\tau$-smoothness, or tightness, which are expressed in terms of $\hat{\mu}$, $\tilde{\mu}$, or $\mu^{\prime}$. (See [5].)

For the reader's convenience, in this part we collect thedefinitions of $\hat{\mu}$, $\tilde{\mu}$, and $\mu^{\prime}$ and we summarize (in the form of a theorem) the principal facts pertaining to the criteria mentioned above.

Preliminaries. Consider any set $X$ and any lattice of subsets of $X, L$, such that $L$ is separating and disjunctive. It is known that the topological space $<\operatorname{IR}(L), \operatorname{tW}(L)>$ is compact and $T_{1}$; it is $T_{2}$ iff $L$ is normal. (See e.g., [4] and [9]). Consider the function $\phi$ which is such that the domain of $\phi$ is $X$ and for every element of $x, x, \phi(x)=\mu_{x}$. Then $\phi$ is $a<t L, t W(L)>$-homeomorphism. For this reason, $\phi(X)$ is topologically identifiable with $X$. Moreover, $\phi(X)$ is dense in $\operatorname{IR}(L)$. Consequently $\operatorname{IR}(L)$ is a compactification of $X$. In case $\phi(X)$ is identified with $X, X$ is said to be embedded in $\operatorname{IR}(L)$.

(i) Definition of $\hat{\mu}$. Denote the general element of $A(L)$ by $A$. Then $\{\mu \in \operatorname{IR}(L) / \mu(A)=1\}$ is denoted by $W(A)$. Moreover, $\{W(L) ; L \epsilon L\}$ is denoted by $W(L)$.

Proposition 1.1.

1. For every element of $A(L), A, W(A)^{\prime}=W\left(A^{\prime}\right)$.

2. For every two elements of $A(L), A, B$,
a) $W(A \cup B)=W(A) \cup W(B)$;
B) $W(A \cap B)=W(A) \cap W(B)$;
$\gamma)$ If $A \supset B$, then $W(A) \supset W(B)$;
ઈ) If $W(A) \supset W(B)$, then $A \supset B$;
ह) $A=B$ iff $W(A)=W(B)$.

3. $A(W(L))=W(A(L))$.

(Note all these statements are true, if $L$ is just disjunctive.) 
Next, consider any element of $M(L), \mu$, and the function $\hat{\mu}$ which is such that the domain of $\hat{\mu}$ is $A(W(L))$ and for every element of $A(W(L)), W(A), \hat{\mu}(W(A))=\mu(A)$. Note $\hat{\mu} \in \mathrm{M}(W(L))$ and if $\mu \in \mathrm{MR}(L)$, then $\hat{\mu} \in \mathrm{MR}(W(L))$. Conversely, consider any element of $M(W(L)), \nu$, and the function $\mu$ which is such that the domain of $\mu$ is $A(L)$ and for every element of $A(L), A, \mu(A)=\nu(W(A))$. Note $\mu<M(L)$ and $\nu=\hat{\mu}$ and if $\nu \in \operatorname{MR}(W(L))$, then $\mu \in \operatorname{MR}(L)$.

Note since $W(L)$ is compact, $\operatorname{MR}(W(L))=\operatorname{MR}(\sigma, W(L))=\operatorname{MR}(\tau, W(L))=\operatorname{MR}(t, W(L))$.

Next, consider any element of $M R(L), \mu$. Then $\hat{\mu} \in \operatorname{MR}(W(L))=M R(\sigma, W(L))$. Hence $\hat{\mu}$ is extendible to the $\sigma$-algebra of $\hat{\mu}^{*}$-measurable sets, uniquely, and the extension is $\delta W(L)$-regular. Continue to use $\hat{\mu}$ for this extension.

(ii) Definition of $\mu^{\prime}$. Denote the general element of $A(L)$ by $A$. Then $\{\mu \in \operatorname{IR}(\sigma, L) / \mu(A)=1\}$ is denoted by $W_{\sigma}(A)$. Moreover, $\left\{W_{\sigma}(L) ; L \in L\right\}$ is denoted by $W_{\sigma}(L)$.

REMARK. If, in each statement of Proposition $1.1, W$ is replaced by $W_{\sigma}$, the resulting statement is true.

Next, consider any element of $M(L), \mu$, and the function $\mu^{\prime}$ which is such that the domain of $\mu^{\prime}$ is $A\left(W_{\sigma}(L)\right)$ and for every element of $A\left(W_{\sigma}(L)\right), W_{\sigma}(A), \mu^{\prime}\left(W_{\sigma}(A)\right)$ $=\mu(A)$. Note $\mu^{\prime} \in M\left(W_{\sigma}(L)\right)$ and if $\mu \in M R(L)$, then $\mu^{\prime} \in \operatorname{MR}\left(W_{\sigma}(L)\right)$. Conversely, consider any element of $M\left(W_{\sigma}(L)\right), \rho$, and the function $\mu$ which is such that the domain of $\mu$ is $A(L)$ and for every element of $A(L), A, \mu(A)=\rho\left(W_{\sigma}(A)\right)$. Note $\mu \in M(L)$ and $\rho=\mu^{\prime}$ and if $\rho \in \operatorname{MR}\left(W_{\sigma}(L)\right)$, then $\mu \in \operatorname{MR}(L)$. Moreover, if $\mu \in \operatorname{MR}(L)$, then $\mu \epsilon \operatorname{MR}(\sigma, L)$ iff $\mu^{\prime} \epsilon \operatorname{MR}\left(\sigma, W_{\sigma}(L)\right)$.

(iii) Definition of $\tilde{\mu}$.

Lemma 1.1. Consider any set $X$ and any two lattices of subsets of $x, L_{1}, L_{2}$, such that $L_{1} \subset L_{2}$. For every element of $\operatorname{MR}\left(L_{1}\right), \mu_{1}$, there exists an element of $\operatorname{MR}\left(L_{2}\right), \mu_{2}$, such that $\left.\mu_{2}\right|_{A\left(L_{1}\right)}=\mu_{1}$ and if $L_{1}$ separates $L_{2}$, then $\mu_{2}$ is unique. (See [2].)

Next, consider any set $X$ and any lattice of subsets of $X, L$, such that $L$ is disjunctive. Consider any element of $\operatorname{MR}(L), \mu$. Then $\hat{\mu} \in \operatorname{MR}(W(L))$. Hence, by Lemma 1.1, there exists an element of $\operatorname{MR}(\operatorname{tW}(L)), \tilde{\mu}$, such that $\left.\tilde{\mu}\right|_{A(W(L))}=\hat{\mu}$ and since $W(L)$ separates $t W(L)$, because $W(L)$ is compact, $\tilde{\mu}$ is unique.

Note since $\operatorname{tW}(L)$ is compact, $\operatorname{MR}(t W(L))=\operatorname{MR}(\sigma, t W(L))=\operatorname{MR}(\tau, t W(L))=\operatorname{MR}(t, t W(L))$.

Consequently, $\tilde{\mu} \in \operatorname{MR}(\sigma, \operatorname{tW}(L))$. Hence $\tilde{\mu}$ is extendible to the $\sigma$-algebra of $\tilde{\mu}^{*}-$ measurable sets, uniquely, and the extension is $t W(L)$-regular. Continue to use $\tilde{\mu}$ for this extension.

THEOREM 1.1. Consider any set $X$ and any lattice of subsets of $X, L$, such that $L$ is. (separating) and disjunctive. For every element of $\operatorname{MR}(L), \mu$ :

1. $\mu \in \operatorname{MR}(\sigma, L)$ iff $\hat{\mu}^{*}(X)=\hat{\mu}(\operatorname{IR}(L))$; equivalently, $\mu \in \operatorname{MR}(\sigma, L)$ iff $\hat{\mu} *(\operatorname{IR}(\sigma, L))=\hat{\mu}(\operatorname{IR}(L))$. 
2. $\mu \in \operatorname{MR}(\tau, L)$ iff $\tilde{\mu}^{*}(X)=\tilde{\mu}(\operatorname{IR}(L))$.

3. $\mu^{\prime} \in \operatorname{MR}\left(\tau, W_{\sigma}(L)\right)$ iff $\tilde{\mu}^{*}(\operatorname{IR}(\sigma, L))=\tilde{\mu}(\operatorname{IR}(L))$.

4. If $L$ is also separating and normal, or $T_{2}$, then $\mu \in \operatorname{MR}(t, L)$ iff $\tilde{\mu}^{*}(X)=$ $\tilde{\mu}(\operatorname{IR}(L))$ and $X$ is $\tilde{\mu}^{*}$-measurable.

We note, for example, that the statement of part $1, " \mu \in \operatorname{MR}(\sigma, L)$ iff $\hat{\mu}^{*}(X)=$

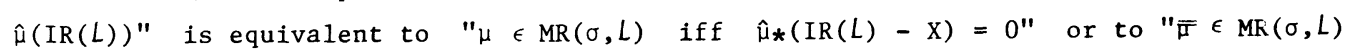
iff for every sequence in $L,\left\langle L_{i}>\right.$, if $\left\langle L_{i}>\right.$ is decreasing and $n_{i} W\left(L_{i}\right) \subset \operatorname{IR}(L)-X_{0}$ then $\hat{\mu}\left(\hat{1} W\left(L_{i}\right)\right)=0 "$. Similarly, equivalent statements are obtainable for the other parts. (For more details refer to [1].)

3. NECESSARY AND SUFFICIENT CONDITIONS.

In this section we work with an arbitrary set $X$ and an arbitrary lattice of subsets of $X, L$, such that $L$ is separating and disjunctive and we give necessary and sufficient conditions for $L$ to be a) Lindelöf, b) replete, c) measure replete, d) strongly measure replete.

a) Lindelöf property.

Theorem 2.1. The following statements are equivalent:

1. $L$ is Lindelöf.

2. For every subset of $L,\left\{L_{\alpha} ; \alpha \in A\right\}$, if $\cap\left\{W\left(L_{\alpha}\right) ; \alpha \in A\right\} \subset I R(L)-X$, then there exists a subset of $A, A^{*}$, such that $\cap\left\{W\left(L_{\alpha}\right) ; \alpha \in A^{*}\right\} \subset \operatorname{IR}(L)-X$ and $A^{*}$ is countable.

Proof. a) Assume 1, and show 2. Consider any subset of $L,\left\{L_{\alpha} ; \alpha \in A\right\}$, such that $n\left\{W\left(L_{\alpha}\right) ; \alpha \in A\right\} \subset \operatorname{IR}(L)-X$. Since $L$ is disjunctive, $n\left\{L_{\alpha} ; \alpha \in A\right\}=\emptyset$.

Hence, since $L$ is Lindelöf, there exists a subset of $A, A^{*}$, such that $n_{\left\{L_{\alpha} ; \alpha \in A\right\}}$ $=\emptyset$ and $A^{*}$ is countable. Consider any such $A^{*}$. Then $n\left\{W\left(L_{\alpha}\right) ; \alpha \in A^{*}\right\} \subset \operatorname{IR}(L)-X$. Consequently 2 is true.

B) Conversely, assume 2, and show 1. (Proof omitted.)

Corollary 2.1. Assume $L$ is normal and countably paracompact. Then the following statements are equivalent:

1. $L$ is Lindelöf.

2. For every element of $t W(L), K$, if $K \subset I R(L)-X$, then there exists an element of $Z(t W(L)), K_{0}$, such that $K \subset K_{0} \subset \operatorname{IR}(L)-X$.

Proof. a) Assume 1, and show 2. Consider any element of $t W(L), K$, such that $K \subset \operatorname{IR}(L)-X$. Since $K \in \operatorname{tW}(L)$, there exists a subset of $L,\left\{L_{\alpha} ; \alpha \in A\right\}$, such that $K=n\left\{W\left(L_{\alpha}\right) ; \alpha \in A\right\}$. Consider any such $\left\{L_{\alpha} ; \alpha \in A\right\}$. Then $n\left\{W\left(L_{\alpha}\right) ; \alpha \in A\right\} \subset \operatorname{IR}(L)-X$. Hence, since $L$ is Lindelof, by Theorem 2.1, there exists a subset of $A, A^{*}$, such that $n\left\{W\left(L_{\alpha}\right) ; \alpha \in A^{*}\right\} \subset \operatorname{IR}(L)-X$ and $A^{*}$ is countable. Consider any such $A^{*}$. Since $L$ is normal and countably paracompact, by [5], Theorem 2.2, part 2, there exists an element of $Z(t W(L)), K_{0}$, such that $n\left\{W\left(L_{\alpha}\right) ; \alpha \in A^{*}\right\} \subset K_{0} \subset \operatorname{IR}(L)-X$. Consider any such $K_{0}$. Then $K \subset K_{0} \subset I R(L)-X$. Consequently 2 is true.

B) Conversely, assume 2, and show 1. Consider any subset of $L,\left\{L_{\alpha} ; \alpha \in A\right\}$, such that $n\left\{\mathrm{~W}\left(\mathrm{~L}_{\alpha}\right) ; \alpha \in \mathrm{A}\right\} \subset \operatorname{IR}(L)-\mathrm{X}$. Set $n\left\{\mathrm{~W}\left(\mathrm{~L}_{\alpha}\right) ; \alpha \in \mathrm{A}\right\}=\mathrm{K}$. Then $\mathrm{K} \subset \operatorname{IR}(L)-\mathrm{X}$. 
Then, since 2 is true, there exists an element of $Z(t W(L)), K_{0}$, such that $K \subset K_{0} \subset$ $\operatorname{IR}(L)-X$. Consider any such $K_{0}$. Then, since $K_{0}$ is $t W(L)$-compact and a $G_{\delta}$-set of $t W(L)$, there exists a sequence in $L,\left\langle L_{n}>\right.$ such that $K_{0}=n\left\{W\left(L_{n}\right)^{\prime} ; n \in N\right\}$. Consider any such $\left\langle L_{n}>\right.$. Then $n\left\{W\left(L_{\alpha}\right) ; a \in A\right\} \subset n\left\{W\left(L_{n}\right)^{\prime} ; n \in N\right\}$. Hence for every $n$, $n\left[W\left(L_{\alpha}\right) ; \alpha \in A\right\} \cap W\left(L_{n}\right)=\emptyset$; hence, since $W(L)$ is compact, there exists an element of $A, \alpha_{n}$, such that $W\left(L_{\alpha_{n}}\right) \subset W\left(L_{n}\right)^{\prime} ;$; consider any such $\alpha_{n}$. Then $\left\{L_{\alpha_{n}} ; n \in N\right\} \subset$ $\left\{L_{\alpha} ; \alpha \in A\right\}$ and $n\left\{W\left(L_{\alpha}\right) ; n \in N\right\} \subset n\left\{W\left(L_{n}\right)^{\prime} ; n \in N\right\}=K_{0} \subset \operatorname{IR}(L)-X$. Then, by Theorem 2.1, $L$ is Linde18f.

Examples. (1). Consider any topological space $X$ such that $X$ is $T_{3 \frac{1}{2}}$ and let $L=2$. Then, by Corollary $2.1, X$ is Lindelyf iff for every closed subset of $B X-X, K$, there exists a zero set of $B X, K_{0}$, such that $K \subset K_{0} \subset B X-X$. (This result is wellknown).

(2). Consider any topological space $X$ such that $X$ is $T_{1}$ and 0 -dimensional and let $L=C$. Then, by Corollary 2.1, $X$ is Lindelof iff for every closed subset of $B_{0} X-X, K$, there exists a zero set of $B_{0} X, K_{0}$, such that $K \subset K_{0} \subset B_{0} X-X$.

b) Repleteness.

Lemma 2.1. Consider any lattice of subsets of $X, L$, such that $L$ is $\delta$. For every element of $\operatorname{MR}(L), \mu$, the following statements are equivalent:

1. $\mu \in \operatorname{MR}(\tau, L)$.

2. For every net in $L,\left\langle\mathrm{~L}_{\alpha}\right\rangle$, if $\left\langle\mathrm{L}_{\alpha}>\right.$ is decreasing, then $\mu^{*} \underset{\alpha}{\left(\cap L_{\alpha}\right)}=$ $\inf _{\alpha} \mu\left(L_{\alpha}\right)$

3. For every subset of $L,\left\{L_{\alpha} ; \alpha \in A\right\}$, if $\left\{L_{\alpha} ; \alpha \in A\right\}$ is a filter base, then $\mu^{*}\left(\cap L_{\alpha}\right)=\inf _{\alpha} \mu\left(L_{\alpha}\right)$. (See [8].)

Theorem 2.2. The following statements are equivalent:

1. $L$ is replete.

2. For every element of $\operatorname{IR}(L), \mu$, if $\mu \in \operatorname{IR}(L)-X$, then there exists an element of $\sigma(W(L)), B$, such that $\mu \in B \subset \operatorname{IR}(L)-X$.

Proof. $\alpha)$ Assume 1, and show 2. Assume $\operatorname{IR}(L)-X \neq \emptyset$ and consider any element of $\operatorname{IR}(L)-X, \mu$. Then $\mu \notin X$. Since $L$ is replete, $\operatorname{IR}(\sigma, L)=X$. Consequently $\mu \notin \operatorname{IR}(\sigma, L)$. Hence there exists a sequence in $L,\left\langle L_{i}\right\rangle$, such that $\left\langle L_{i}\right\rangle$ is decreasing and $\lim _{i} L_{i}=\emptyset$, but $\lim _{i} \mu\left(L_{i}\right) \neq 0$. Consider any such $\left\langle L_{i}>\right.$. Note for every i, $\mu\left(L_{i}\right)=1$. Consequent1y $\mu \in \underset{i}{n} W\left(L_{i}\right) \subset \operatorname{IR}(L)-X$ and ${ }_{i} W\left(L_{i}\right) \epsilon \sigma(W(L))$. Consequently 2 is true.

B) Conversely, assume 2, and show 1 . Note to show $L$ is replete, it suffices to show $\operatorname{IR}(\sigma, L)-X=\emptyset$. Assume $\operatorname{IR}(\sigma, L)-X \neq \emptyset$. Consider any element of $\operatorname{IR}(\sigma, L)-X$, $v$. Then $\nu \in \operatorname{IR}(L)-X$. Hence, since 2 is true, there exists an element of $\sigma(W(L))$, $B$, such that $\nu \in B \subset \operatorname{IR}(L)-X$. Consider any such $B$.

(i) Since $\nu \in \mathbb{M R}(L)$, the extension of $\hat{v}$ to the $\sigma$-algebra of $\hat{v}^{*}$-measurable sets (also denoted by $\hat{v}$ ) is $\delta W(L)$-regular. Consequently $\hat{v}(B)=\sup \{\hat{v}(K) / K \epsilon \delta W(L)$ 
and $K \subset B\}$. Consider any element of $\delta W(L)$, $K$, such that $K \subset B$. Then $K \subset \operatorname{IR}(L)$ $-X$. Hence, by Theorem 1.1 , part $1, \hat{v}(K)=0$. Consequently $\hat{v}(B)=0$.

(ii) Since $\nu \in \operatorname{IR}(L),\{v\}=n\{W(L) / L \in L$ and $\hat{v}(W(L))=1\}$. Hence, by Lemma $2.1, \hat{v}^{*}(\{v\})=1$.

(iii) Consequently $1=\hat{v}^{*}(\{\nu\}) \leq \hat{v}(B)=0$. Thus a contradiction has been reached. Consequently $\operatorname{IR}(\sigma, L)-X=\emptyset$, and $L$ is replete.

COROLLARY 2.2. $L$ is replete iff whenever $\mu \in \operatorname{IR}(L)-X$, then there exists a

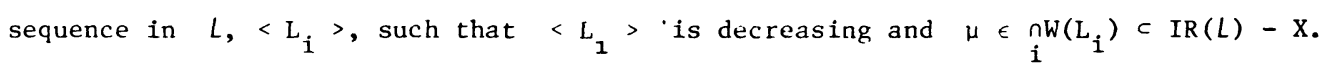

(Proof omitted.)

Example. Consider any topological space $X$ such that $X$ is $T_{1}$ and let $L=F$. Then, by Corollary 2.2, $X$ is $\alpha$-complete iff whenever $\mu \in \omega X-X$, then there exists a sequence in $F,\left\langle F_{i}\right\rangle$, such that $\left\langle F_{i}\right\rangle$ is decreasing and $\mu \epsilon \underset{i}{n} \bar{F}_{i} \subset \omega X-X$, where the closure is taken in $\omega X$.

COROELARY 2.3. Assume $L$ is normal and countably paracompact. Then the following statements are equivalent:

1. $L$ is replete.

2. For every element of $\operatorname{IR}(L), \mu$, if $\mu \in \operatorname{IR}(L)-X$, then there exists an element of $Z(t W(L)), K_{0}$, such that $\mu \in \mathrm{K}_{0} \subset \operatorname{IR}(L)-\mathrm{X}$.

Proof. a) Assume 1, and show 2. Assume $\operatorname{IR}(L)-X \neq \emptyset$ and consider any element of $I R(L)-X, \mu$. Then, since $L$ is replete, by Corollary 2.2, there exists a sequence in $L,\left\langle L_{i}\right\rangle$, such that $\left\langle L_{i}>\right.$ is decreasing and $\mu \epsilon \underset{i}{n W}\left(L_{i}\right) \subset \operatorname{IR}(L)-X$. Consider any such $\left\langle\mathrm{L}_{i}>\right.$. Then, since $L$ is normal and countably paracompact, by [5], Theorem 2.2, part 2, there exists an element of $Z(t W(L)), K_{0}$, such that $n_{i} W\left(L_{i}\right) \subset k_{0} \subset$ $I R(L)$ - X. Consider any such $K_{0}$. Then $\mu \in K_{0} \subset \operatorname{IR}(L)-X$. Consequently 2 is true.

B) Conversely, assume 2, and show 1. To show $L$ is replete, use Corollary 2.2. Assume $\operatorname{IR}(L)-X \neq \emptyset$ and consider any element of $\operatorname{IR}(L)-X, \mu$. Then, since 2 is true, there exists an element of $Z(t W(L)), K_{0}$, such that $\mu \epsilon K_{0} \subset \operatorname{IR}(L)-X$. Consider any such $K_{0}$. Then, since $K_{0}$ is a $G_{\delta}$-set of $t W(L)$, there exists a sequence in $\mathrm{tW}(\mathrm{L}),\left\langle\mathrm{H}_{i}\right\rangle$, such that $\mathrm{K}_{0}=\underset{i}{\mathrm{nH}_{i}^{\prime}}$. Consider any such $\left\langle\mathrm{H}_{i}\right\rangle$. Then for every $i, K_{0} \cap H_{i}=\emptyset$; hence, since $W(L)$ separates $t W(L)$, there exist two elements of $L, L_{i}, \tilde{L}_{i}$, such that $K_{0} \subset W\left(L_{i}\right)$ and $H_{i} \subset W\left(\tilde{L}_{i}\right)$ and $W\left(L_{i}\right) \cap W\left(\tilde{L}_{i}\right)=\emptyset$; consider any such $L_{i}, \tilde{L}_{i}$; then $k_{0} \subset W\left(L_{i}\right) \subset W\left(\tilde{L}_{i}\right)^{\prime} \subset H_{i}^{\prime}$. Consequently $K_{0} \subset \underset{i}{n W}\left(L_{i}\right) \subset \underset{i}{n} H_{i}^{\prime}=$ $\mathrm{K}_{0}$. Hence $\mathrm{K}_{0}=\mathrm{nW}_{i}\left(\mathrm{~L}_{i}\right)$. Without loss of generality, assume that $\left\langle\mathrm{L}_{i}>\right.$ is decreas-

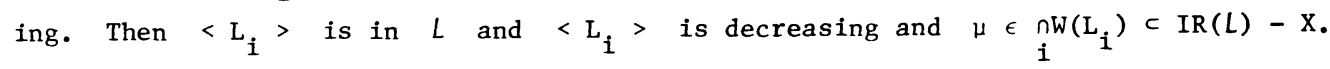
Hence, by Corollary $2.2, L$ is replete.

Examples. (1). Consider any topological space $X$ such that $X$ is $T_{3 \frac{1}{2}}$ and let $L=Z$. Then, by Corollary 2.3, $X$ is realcompact iff whenever $\mu \in \beta X-X$, then there exists a zero set of $\beta X, K_{0}$, such that $\mu \in K_{0} \subset \beta X-X$. (This special case is known.) 
(2). Consider any topological space $X$ such that $X$ is $T_{1}$ and 0-dimensional and let $L=C$. Then, by Corollary 2.3, $X$ is $N$-compact iff whenever $\mu \epsilon B_{0} X-X$, then there exists a zero-set of $B_{0} X, K_{0}$, such that $\mu \in K_{0} \subset B_{0} X-X$.

COROLLARY 2.4. If there exists a collection of $F_{\sigma}$-sets of $t W(L),\left\{H_{\alpha} ; \alpha \in A\right\}$, such that $\mathrm{X}=\mathrm{N}_{\alpha}^{\mathrm{H}} \mathrm{C}_{\alpha}$, then $L$ is replete.

Proof. Assume there exists a collection of $F_{\sigma}$-sets of $t W(L),\left\{H_{\alpha} ; \alpha \in A\right\}$, such that $\mathrm{X}=\mathrm{NH}_{\alpha}$. Consider any such $\left\{\mathrm{H}_{\alpha} ; \alpha \in A\right\}$. To show $L$ is replete, use Theorem 2.2. Assume $\operatorname{IR}(L)-X \neq \emptyset$ and consider any element of $\operatorname{IR}(L)-X$, $\mu$. Since $\mathrm{X}=\mathrm{n}_{\alpha}^{\mathrm{n}} \mathrm{H}, \operatorname{IR}(L)-\mathrm{X}=\mathrm{U}_{\alpha}^{\prime} \mathrm{H}_{\alpha}^{\prime}$. Consequently there exists an element of $A, \alpha_{0}$, such that $\mu \in \mathrm{H}_{\alpha_{0}}^{\prime}$. Consider any such $\alpha_{0}$. Then, since $\mathrm{H}_{\alpha_{0}}$ is an $F_{\sigma}$-set of $\operatorname{tw}(L), H_{\alpha_{0}}^{\prime}$ is a $G_{\delta}$-set of $t W(L)$. Then, since $\mu \in H_{\alpha_{0}}^{\prime}$, there exists a sequence in $L,\left\langle L_{i}>\right.$, such that $\mu \in \underset{i}{n W}\left(L_{i}\right) \subset H_{\alpha_{0}}^{\prime}$. (See the proof of part $\beta$ ) of Corollary 2.3.) Consider any such $\left\langle L_{i}>\right.$. Then $n_{i} W\left(L_{i}\right) \in \sigma(W(L))$ and $\mu \in \underset{i}{n} W\left(L_{i}\right) \subset I R(L)-X$. Hence, by Theorem $2.2, L$ is replete.

COROLLARY 2.5. If there exists a subset of $Z(t W(L)),\left\{K_{\alpha} ; \alpha \in A\right\}$, such that $\mathrm{X}=\mathrm{nK}_{\alpha}^{\prime}$, then $L$ is replete.

Proof. Assume there exists a subset of $Z(t W(L)),\left\{K_{\alpha} ; \alpha \in A\right\}$, such that $X=\mathrm{XK}_{\alpha}^{\prime}$. Consider any such $\left\{\mathrm{K}_{\alpha} ; \alpha \in \mathrm{A}\right\}$. Note for every $\alpha$, since $\mathrm{K}_{\alpha} \in Z(\mathrm{tW}(L)), \mathrm{K}_{\alpha}$ is a $G_{\delta}$-set of $\operatorname{tW}(L)$. Hence for every $\alpha, K_{\alpha}^{\prime}$ is an $F_{\sigma}$-set of $t W(L)$. Then, by Corollary $2.4, L$ is replete.

c) Measure repleteness.

Observation. Note for every element of $\operatorname{IR}(L), \mu, \mu \epsilon \operatorname{IR}(\sigma, L)$ iff $\mu^{\prime} \epsilon \operatorname{IR}(\sigma$, $\left.W_{\sigma}(L)\right)$. Next, for the general element of $\operatorname{IR}\left(\sigma, W_{\sigma}(L)\right), \mu^{\prime}$, note $S\left(\mu^{\prime}\right)=\cap\left\{W_{\sigma}(L) / L \epsilon L\right.$ and $\mu^{\prime}\left(W_{\sigma}(L)\right)=1$ \}. Consider any element of $L, L$, such that $\mu^{\prime}\left(W_{\sigma}(L)\right)=1$. Then, by the definition of $\mu^{\prime}, \mu(L)=1$. Consequently $\mu \in W_{\sigma}(L)$. Hence $\mu \in S\left(\mu^{\prime}\right)$, so $S\left(\mu^{\prime}\right) \neq \emptyset$. Consequently $W_{\sigma}(L)$ is replete.

Summarizing: If $L$ is disjunctive, then $W_{\sigma}(L)$ is replete.

We will obtain a necessary and sufficient condition for $W_{\sigma}(L)$ to be measure replete.

Preliminaries: Consider the set whose general element is an element of $\operatorname{MR}(L), \mu$, such that $\mu^{\prime} \in \operatorname{MR}\left(\tau, W_{\sigma}(L)\right)$. This set is denoted by $\hat{M R}(L)$. (See [5], p. 1517.)

According to [5], Theorem 3.2, part $1, \operatorname{MR}(L) \subset \operatorname{MR}(\sigma, L)$.

Theorem 2.3. The following statements are equivalent:

1. $\mathrm{W}_{\sigma}(L)$ is measure replete.

2. $\hat{\operatorname{MR}}(L)=\operatorname{MR}(\sigma, L)$.

Proof. $\alpha)$ Assume 1, and show 2. Note to show $\hat{\operatorname{MR}}(L)=\operatorname{MR}(\sigma, L)$, it suffices to show $\operatorname{MR}(\sigma, L)=\hat{\operatorname{MR}}(L)$. Consider any element of $\operatorname{MR}(\sigma, L), \mu$. Then $\mu^{\prime} \in \operatorname{MR}\left(\sigma, W_{\sigma}(L)\right)$. 
Since $W_{\sigma}(L)$ is measure replete, by assumption, $\operatorname{MR}\left(\sigma, W_{\sigma}(L)\right)=M R\left(\tau, W_{\sigma}(L)\right)$. Consequently $\mu^{\prime} \in \operatorname{MR}\left(\tau, W_{\sigma}(L)\right)$, so $\mu \in \hat{M R}(L)$. Hence $\operatorname{MR}(\sigma, L) \subset \hat{M R}(L)$. Consequently, $\hat{\operatorname{MR}}(L)=\operatorname{MR}(\sigma, L)$.

B) Converse1y, asisume 2, and show 1. (Proof omitted.)

COROLLARY 2.6. The following statements are equivalent:

1. $L$ is measure replete.

2. $L$ is replete and $\hat{M R}(L)=\operatorname{MR}(\sigma, L)$.

Proof. a) Assume 1 , and show 2. Since $L$ is measure replete, $L$ is replete. Hence $\operatorname{IR}(\sigma, L)=X$. Consequently, $W_{\sigma}(L)=L$. Hence, since $L$ is measure replete, by assumption, $W_{\sigma}(L)$ is measure replete. Then, by Theorem 2.3, $\hat{M R}(L)=\operatorname{MR}(\sigma, L)$. Consequently 2 is true.

B) Conversely, assume 2, and show 1. (Proof omitted).

Examples. (1). Consider any topological space $X$ such that $X$ is $T_{3 \frac{1}{2}}$ and let $L=Z$. Then, by Corollary 2.6, $X$ is measure compact iff $X$ is realcompact and $\hat{\operatorname{MR}}(Z)=\operatorname{MR}(\sigma, Z)$.

(2). Consider any topological space $X$ such that $X$ is $T_{1}$ and let $L=F$. Then, by Corollary 2.6, $\mathrm{X}$ is Borel measure compact iff $\mathrm{X}$ is $\alpha$-complete and $\hat{M R}(F)=$ $\operatorname{MR}(\sigma, F)$.

(3). Consider any topological space $X$ such that $X$ is $T_{1}$ and 0 -dimensional and let $L=C$. Then, by Corollary $2.6, X$ is clopen measure replete iff $X$ is $N-$ compact and $\hat{\operatorname{MR}}(\mathcal{C})=\operatorname{MR}(\sigma, \mathcal{C})$.

Lemma 2.2. For every element of $\operatorname{MR}(L), \mu$, for every element of $t W(L), K$, $\hat{\mu}^{*}(\mathrm{~K})=\tilde{\mu}(\mathrm{K})$.

Proof. Consider any element of $\operatorname{MR}(L), \mu$, and any element of $t W(L), K$. Since $K \in t W(L), K=n\{W(L) / L \in L$ and $W(L)>K\}$. Set $\{W(L) / L \in L$ and $W(L) \supset K\}=$ $\left\{W\left(L_{\alpha}\right) ; \alpha \in A\right\}$. Note $\delta W(L)$ is $\delta$ and $\hat{\mu} \in \operatorname{MR}(\tau, \delta W(L))$ and $\left\{W\left(L_{\alpha}\right) ; \alpha \in A\right\} \subset \delta W(L)$

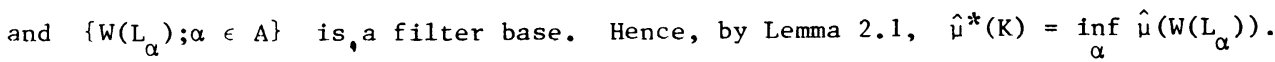
Since $\left.\tilde{\mu}\right|_{A(W(L))}=\hat{\mu}$. $\inf _{\alpha} \hat{\mu}\left(W\left(L_{\alpha}\right)\right)=\inf _{\alpha} \tilde{\mu}\left(W\left(L_{\alpha}\right)\right)$. Now, note $t W(L)$ is $\delta$ and $\tilde{\mu} \in \operatorname{MR}(\tau, \mathrm{tW}(L))$ and $\left\{\mathrm{W}\left(\mathrm{L}_{\alpha}\right) ; \alpha \in \mathrm{A}\right\} \subset \mathrm{tW}(L)$ and $\left\{\mathrm{W}\left(\mathrm{L}_{\alpha}\right) ; \alpha \in \mathrm{A}\right\}$ is a filter base. Hence, by Lemma 2.1 , $\inf _{\alpha} \tilde{\mu}\left(W\left(L_{\alpha}\right)\right)=\tilde{\mu}(K)$. Consequently $\hat{\mu}^{*}(K)=\tilde{\mu}(K)$.

Remark. The condition " $L$ is separating and disjunctive" was not needed in the proof.

Observation. For every element of $\operatorname{MR}(L), \mu, \hat{\mu}^{*} \geq \tilde{\mu}^{*}$. (Proof omitted.)

THEOREM 2.4. The following statements are equivalent:

1. $L$ is measure replete.

2. For every element of $M R(\sigma, L), \mu$, for every element of $t W(L), K$, if $K$. $\operatorname{IR}(L)-\mathrm{X}$, then $\hat{\mu}^{*}(\mathrm{~K})=0$.

Proof. a) Assume 1, and show 2. Consider any element of $\operatorname{MR}(\sigma, L), \mu$, and any element of $t W(L), K$, such that $K \subset \operatorname{IR}(L)-X$. Since $K \in \operatorname{tW}(L)$ and $\mu \in \operatorname{MR}(L)$, by Lemma 2.2, $\hat{\mu}^{*}(K)=\tilde{\mu}(K)$. Since $L$ is measure replete, by assumption, $\operatorname{MR}(\sigma, L) \subset$ $\operatorname{MR}(\tau, L)$. Consequently $\mu \in \operatorname{MR}(\tau, L)$. Hence, since $K \in \operatorname{tW}(L)$ and $K \subset \operatorname{IR}(L)-X$, by Theorem 1.1, part 2, $\tilde{\mu}(K)=0$. Consequently $\hat{\mu}^{*}(K)=0$. Thus 2 is true. 
B) Conversely, assume 2, and show 1. Note to show $L$ is measure replete, it suffices to show $\operatorname{MR}(\sigma, L) \subset \operatorname{MR}(\tau, L)$. Consider any element of $\operatorname{MR}(\sigma, L), \mu$. To show $\mu \in \operatorname{MR}(\tau, L)$, use Theorem 1.1, part 2. Consider any element of $t W(L), K$, such that $\mathrm{K} \subset \mathrm{IR}(L)-\mathrm{X}$. Since $\mathrm{K} \in \operatorname{tW}(L)$ and $\mu \in \operatorname{MR}(L)$, by Lemma $2.2, \hat{\mu}^{*}(\mathrm{~K})=\tilde{\mu}(\mathrm{K})$. Since $K \in \operatorname{tW}(L)$ and $K \subset \operatorname{IR}(L)-X$ and $\mu \in \operatorname{MR}(\sigma, L)$, by the assumption, $\hat{\mu}^{*}(K)=0$. Consequently $\tilde{\mu}(K)=0$. Then, by Theorem 1.1, part 2, $\mu \in \operatorname{MR}(\tau, L)$. Hence $\operatorname{MR}(\sigma, L) \subset$ $\operatorname{MR}(\tau, L)$. Consequently $L$ is measure replete.

Remark. In this connection, we note the following useful result:

Proposition 2.1. Consider any two lattices of subsets of $x, L_{1}, L_{2}$, such that $L_{1} \subset L_{2}$ and any element of $\operatorname{MR}\left(\sigma, L_{2}\right), v$, such that $\left.v\right|_{A\left(L_{1}\right)} \in \operatorname{MR}\left(\sigma, L_{1}\right)$. Set $\left.v\right|_{\left.A \mid L_{l}\right)}=\mu$. Then $\mu^{*}=\nu^{*}$ iff $\mu^{*}=\nu$ on $L_{2}^{\prime}$.

(Proof omitted.)

COROLLARY $(\alpha)$. For every element of $\operatorname{MR}(L), \mu, \hat{\mu}^{*}=\tilde{\mu}^{*}$ iff $\hat{\mu}^{*}=\tilde{\mu}$ on $t W(L)$. Proof. Consider any element of $\operatorname{MR}(L), \mu$. Now, use Proposition 2.1 with $L_{1}=$ $W(L), \quad L_{2}=t W(L), \quad v=\tilde{\mu}$.

COROLLARY $(B)$. For every element of $\operatorname{MR}(L), \mu$, if $\hat{\mu}^{*}=\tilde{\mu}$ on $t W(L)^{\prime}$, then $\mu \in \operatorname{MR}(\sigma, L)$ implies $\mu \in \operatorname{MR}(\tau, L)$.

(Proof omitted).

COROLLARY 2.7. If for every element of $t W(L), K, K \subset \operatorname{IR}(L)-X$ implies there exists an element of $\sigma(W(L)), B$, such that $K \subset B \subset \operatorname{IR}(L)-X$, then $L$ is measure replete.

Proof. Assume for every element of $t W(L), K, K \subset \operatorname{IR}(L)-X$ implies there exists an element of $\sigma(W(L)), B$, such that $K \subset B \subset \operatorname{IR}(L)-X$. To show $L$ is measure replete, use Theorem 2.4. Consider any element of $\operatorname{MR}(\sigma, L), \mu$, and any element of $t W(L), K$, such that $K \subset I R(L)-X$. Then, by the assumption, there exists an element of $\sigma(W(L)), B$, such that $K \subset B \subset \operatorname{IR}(L)-X$. Consider any such B. Then $\hat{\mu}^{*}(K) \leq \hat{\mu}(B)$. Moreover, since $\mu \in \operatorname{MR}(\sigma, L)$ and $B \in \sigma(W(L))$ and $B \subset \operatorname{IR}(L)-X$, $\hat{\mu}(B)=0$. (See the proof of Theorem 2.2, part $B) . \quad$ Consequently $\hat{\mu}^{*}(K)=0$. Then by. Theorem $2.4, L$ is measure replete.

REMARK. Corollary 2.7 is the measure repleteness analog of Theorem 2.2 for repleteness.

Observation. Since, in general, $Z(t W(L)) \subset \sigma(W(L)), \sigma(Z(t W(L)), \subset \sigma(W(L))$. (Note $\sigma(Z(t W(L)))$ is the class of Baire sets of $\operatorname{IR}(L)$.

Examples. (1). Consider any topological space $X$ such that $X$ is $T_{3 \frac{1}{2}}$ and let $L=Z$. If for every closed subset of $B X, K, K \subset B X-X$ implies there exists a Baire set of $B X, B$, such that $K \subset B \subset B X-X$, then, by Corollary 2.7, $X$ is measure compact.

(2). Consider any topological space $X$ such that $X$ is $T_{1}$ and let $L=F$. If for every closed subset of $\omega X, K, K \subset \omega X-X$ implies there exists a Baire set of $\omega X, B$, such that $K \subset B \subset \omega X-X$, then, by Corollary 2.7, $X$ is Borel measure compact.

(3). Consider any topological space $X$ such that $X$ is $T_{1}$ and 0 -dimensional and let $L=E$. If for every closed subset of $\beta_{0} X, K, \ldots \subset^{\circ} \beta_{0} X-X$ implies there exists a Baire set of $\beta_{0} X, B$, such that $K^{\subset}{ }_{B} \subset \beta_{0} X-X$, then, by Corollary 2.7 , $X$ is clopen measure replete. 
THEOREM 2.5. The following statements are equivalent:

1. $L$ is measure replete.

2. For every element of $\operatorname{MR}(\sigma, L), \mu$, for every subset of $L,\left\{L_{\alpha} ; \alpha \in A\right\}$, if $n\left\{L_{\alpha} ; \alpha \in A\right\}=\emptyset$, then there exists a subset of $A, A^{*}$, such that $A^{*}$ is countable and $\mu\left(n\left\{\mathrm{~L}_{\alpha} ; \alpha \in \mathrm{A}^{\star}\right\}\right)=0$.

(Proof omitted.)

REMARK. The condition " $L$ is separating and disjunctive" is not needed in the proof of this theorem.

d) Strongly measure repleteness.

THEOREM 2.6. If $L$ is normal (or $T_{2}$ ) and $X \in \sigma(W(L))$, then $L$ is strongly measure replete.

Proof. Assume $L$ is normal (or $T_{2}$ ) and $X \in \sigma(W(L))$. Note to show $L$ is strongly measure replete, it suffices to show $\operatorname{MR}(\sigma, L) \subset \operatorname{MR}(t, L)$. Consider any element of $\operatorname{MR}(\sigma, L), \mu$. To show $\mu \in \mathbb{R}(t, L)$, use Theorem 1.1, part 4. Since $X \epsilon$ $\sigma(W(L)), X$ is $\tilde{\mu}^{*}$-measurable. Moreover, $\tilde{\mu}^{*}(X)=\tilde{\mu}(X)$, since $X$ is $\tilde{n}^{*}$-measurable,

$$
\begin{aligned}
& =\hat{\mu}(X) \text {, since }\left.\tilde{\mu}\right|_{\sigma(W(L))}=\hat{\mu}, \\
& =\hat{\mu}^{*}(X) \text {, since } X \text { is } \hat{\mu}^{*} \text {-measurable, } \\
& =\hat{\mu}(\operatorname{IR}(L)) \text {, since } \mu \in \mathbb{M}(\sigma, L), \\
& =\tilde{\mu}(\operatorname{IR}(L)) \text {. Consequently }
\end{aligned}
$$

$\tilde{\mu}^{*}(X)=\tilde{\mu}(\operatorname{IR}(L))$ and $X$ is $\tilde{\mu}^{*}$-measurable. Then, since $L$ is separating, disjunctive, and normal (or $T_{2}$ ), by Theorem 1.1, part 4, $\mu \in \operatorname{MR}(t, L)$. Hence $\operatorname{MR}(\sigma, L) \subset \operatorname{MR}(t, L)$.

Consequently $L$ is strongly measure replete.

Examples. (1). Consider any topological space $X$ such that $X$ is $T_{3 \frac{1}{2}}$ and 1 et $L=Z$. If $X$ is a Baire set of $B X$, then, by Theorem $2.6, X$ is strongly measure compact.

(2). Consider any topological space $X$ such that $X$ is $T_{3 \frac{1}{2}}$ and normal or simply $\mathrm{T}_{2}$ and let $L=F$. If $\mathrm{X}$ is a Baire set of $\omega X$, then, by Theorem 2.6, $\mathrm{X}$ is strongly Borel measure compact.

(3). Consider any topological space $X$ such that $X$ is $T_{1}$ and 0 -dimensional and let $L=C$. If $x$ is a Baire set of $B_{0} X$, then, by Theorem 2.6, $x$ is strongly clopen measure replete.

4. REPLETENESS PROPERTIES.

It is advantageous to be able to characterize various repleteness properties in terms of support of certain measures. In this section we pursue this matter in general. Consider any set $X$ and any lattice of subsets of $X, L$, such that $L$ is separating and disjunctive.

a) Repleteness and support.

Preliminaries. Consider the set whose general element is an elemenc of $\operatorname{MR}(L), \mu$, such that whenever $\rho \in \operatorname{IR}(L)-\operatorname{IR}(\sigma, L)$, then there exists an element of $\operatorname{tW}(L)^{\prime}, 0$, such that $\rho \in 0$ and $\tilde{\mu}(0)=0$. This set is denoted by $\tilde{M}_{R}(L)$. (See $\left.[1], p_{.} 1519.\right)$ Next, consider the set whose general element is an element of $\operatorname{MR}(L), \mu$, such that whenever $\rho \in \operatorname{IR}(L)-X$, then there exists an element of $t W(L)^{\prime}, 0$, such that $\rho \in 0$ and $\tilde{\mu}(0)=0$. Denote this set by $\breve{M R}(L)$. Note $\check{M} R(L) \neq \emptyset$ and $\breve{M R}(L) \subset \tilde{M} R(L)$. 
LEMMA 3.1. For every element of $\operatorname{MR}(L), \mu, \mu \in \breve{M R}(L)$ iff $S(\tilde{\mu}) \subset \mathrm{X}$.

Proof. $\alpha)$ Consider any element of $\check{I} \mathbb{R}(L), \mu$. Now, consider any element of $S(\tilde{\mu})$, $\rho$. (Note, since $t W(L)$ is compact, $S(\tilde{\mu}) \neq \emptyset$.) Then, since $S(\tilde{\mu}) \subset \operatorname{IR}(L), \rho \in \operatorname{IR}(L)$. Assume $\rho \notin X$. Then $\rho \in \operatorname{IR}(L)-X$. Hence, since $\mu \epsilon \check{M} R(L)$, by the definition of $\operatorname{Mr}(L)$, there exists an element of $t W(L)^{\prime}, 0$, such that $\rho \epsilon 0$ and $a(0)=0$. Consequently, $\rho \notin S(\tilde{\mu})$. Thus a contradiction has been reached. Consequently $S(\tilde{\mu}) \subset \mathrm{X}$.

$B)$ Consider any element of $\operatorname{MR}(L), \mu$, such that $S(\tilde{\alpha}) \subset x$. Assume $\operatorname{IR}(L)-X \neq \emptyset$ and consider any element of $\operatorname{IR}(L)-X, \rho$. Then $\rho \notin S(\tilde{\mu})$. Consequently there exists an element of $t W(L)^{\prime}, 0$, such that $\rho \in 0$ and $\tilde{\mu}(0)=0$. Then, by the definition of $\check{\operatorname{MR}}(L), \quad \mu \in \check{\mathrm{M}} \mathrm{R}(L)$.

COROLLARY 3.1. For every element of $\operatorname{MR}(L), \mu, \mu \in \stackrel{\mathscr{M} R}{\operatorname{Mr}(L)}$ iff $S(\mu)=S(\tilde{\mu})$.

Proof. $\alpha)$ Consider any element of $\check{M}(L), \mu$. Since $\mu \in \mathbb{M R}(L), \tilde{\mu}$ exists and $S(\mu)=S(\tilde{\mu}) \cap X$. Since $\mu \in Y_{R}(L)$, by Lemma 3.1, part $\left.\alpha\right), S(\tilde{\mu}) \subset X$. Consequently $S(\mu)=S(\tilde{\mu})$.

$B)$ Consider any element of $M R(L), \mu$, such that $S(\mu)=S(\tilde{\mu})$. Then, since $S(\mu) \subset X, S(\tilde{\mu}) \subset X$. Then; by Lemma 3.1, part $\beta), \mu \in \stackrel{M}{\operatorname{MR}(L) .}$

COROLLARY 3.2. $\check{M} R(L) \subset \operatorname{MR}(\tau, L)$.

Proof. Consider any element of $\stackrel{M}{M}(L), \mu$. To show $\mu \in \operatorname{MR}(\tau, L)$, use Theorem 1.1, part 2. Consider any element of $t W(L), K$, such that $K \subset \operatorname{IR}(L)-X$. Since $\mu \in \overleftarrow{M} R(L)$, by Lemma 3.1, $S(\tilde{\mu}) \subset X$. Consequent $1 y \quad K \cap S(\tilde{\mu})=\emptyset$. Moreover, since $S(\tilde{\mu})=$ $n\{W(L) / L \in L$ and $\tilde{\mu}(W(L))=\tilde{\mu}(\operatorname{IR}(L))\}$, by Lemma 2.1, $\tilde{\mu}(S(\tilde{\mu}))=\tilde{\mu}(\operatorname{IR}(L))$. Consequently $\tilde{\mu}(K)=0$. Then, by Theorem 1.1, part 2, $\mu \in \operatorname{MR}(\tau, L)$. Hence $\breve{M R}(L) \subset \operatorname{MR}(\tau, L)$.

THEOREM 3.1. $L$ is replete iff $\tilde{M}(L) \subset \check{M}(L)$.

Proof. By [5], Theorem 3.5, part 3, $L$ is replete iff whenever $\mu \in \tilde{M} R(L)$, then $S(\tilde{\mu}) \subset X$. By Lemma 3.1, for every element of $\operatorname{MR}(L), \mu, \mu \in \operatorname{MR}(L)$ iff $S(\tilde{\mu}) \subset X$. Consequent1y $L$ is replete iff $\tilde{M} R(L) \subset \check{M} R(L)$. (Recal1 that, in general, $\check{M}_{R}(L) \subset \tilde{M} R(L)$.) THEOREM 3.2. 1) If $L$ is replete, then for every element of $M(L), \mu, S\left(\mu^{\prime}\right)=$ $S(\mu)$.

2) If for every element of $\operatorname{IR}(\sigma, L), \mu, S\left(\mu^{\prime}\right)=S(\mu)$, then $L$ is replete.

Proof. 1) Assume $L$ is replete. Consider any element of $M(L), \mu$. Then $S\left(\mu^{\prime}\right)=S(\hat{\mu}) \cap \operatorname{IR}(\sigma, L)$. Hence, since $L$ is replete, $S\left(\mu^{\prime}\right)=S(\breve{\mu}) \cap X$. Further, note $S(\hat{\mu}) \cap X=S(\mu)$. Consequently $S\left(\mu^{\prime}\right)=S(\mu)$.

2) Assume for every element of $\operatorname{IR}(\sigma, L), \mu, S\left(\mu^{\prime}\right)=S(\mu)$. To show $L$ is replete, assume the contrary. Then $\operatorname{IR}(\sigma, L)-X \neq \emptyset$. Consider any element of $\operatorname{IR}(\sigma, L)-X, \mu$. Then $S\left(\mu^{\prime}\right)=\{\mu\}$ and $S(\mu)=\emptyset$. Thus a contradiction has been reached. Consequent1y $L$ is replete.

B) Measure repleteness and support.

The purpose of the following example is to show that the condition "there exists an element of $\operatorname{MR}(\sigma, L), \nu$, such that $S(\nu) \neq \emptyset^{\prime \prime}$ is not sufficient for $L$ to be measure replete.

Example. Assume $L$ is not compact. Then $\operatorname{IR}(L)-X \neq \emptyset$.

a) Consider any element of $\operatorname{IR}(L)-X, \mu$, and any element of $x, x$. Then, consider $\mu+\mu_{x}$ and denote it by $\nu$. Since $\mu \in \operatorname{IR}(L)$ and $\mu_{x} \in \operatorname{IR}(L)$ (because $L$ is 
disjunctive), $\nu \in \mathbb{M R}(L)$. By the definition of support, $S(\nu)=n[L \in L / \nu(L)=\nu(X)\}$. Consider any element of $L, L$, such that $\nu(L)=\nu(X)$. Then $\nu(L)=\nu(X)=\mu(X)+\mu(X)$ $=1+1=2$. Consequently $\mu(L)+\mu_{x}(L)=2$. Hence $\mu_{x}(L)=1$, and $x \in L$. Consequently $x \in S(v)$, and $S(v) \neq \emptyset$.

Next, show $\nu \notin \operatorname{MR}(\tau, L)$. Assume $\nu \in \operatorname{MR}(\tau, L)$. Then, since $\mu=\nu-\mu_{x}, \mu \epsilon$ $\operatorname{MR}(\tau, L)$. Consequently $\mu \in \operatorname{IR}(\tau, L)$. Since $L$ is separating and disjunctive, $\operatorname{IR}(\tau, L)=X$. Consequently $\mu \in X$. Thus a contradiction has been reached. Consequent$1 y \vee \mathbb{M R}(\tau, L)$.

B) Assume $L$ is not replete. Then $\operatorname{IR}(\sigma, L) \neq x$. Consider any element of $\operatorname{IR}(\sigma, L)$ $-X, \mu$, any element of $X, x$, and $\nu$ (as in part $\alpha$ ). Then $\nu \in \mathbb{M R}(\sigma, L), S(\nu) \neq \emptyset$, and $\nu \notin \mathbb{M R}(\tau, L)$ (see part $\alpha)$ ). Consequent1y $\nu \in \mathbb{M R}(\sigma, L), S(\nu) \neq \emptyset$, but $L$ is not measure replete.

Observation. For every element of $\operatorname{IR}(\sigma, L), \nu$, if $S(\nu) \neq \emptyset$, then $\nu \in \operatorname{IR}(\tau, L)$. We will give a necessary and sufficient condition for measure repleteness in terms of support.

LEMMA 3.2. Consider any set $X$ and any lattice of subsets of $X$, $L$. Consider any element of $\operatorname{MR}(L), \mu$, and the measures $\hat{\mu}$ on $\sigma(W(L))$ and $\hat{p}$ on $\sigma(t W(L))$. (Recall $\hat{\mu}$ is $\delta W(L)$-regular and $\hat{\jmath}$ is $t W(L)$-regular.) Next, consider any subset of $\operatorname{IR}(L)$, H. Then

Case 1: There exists a countably additive measure on $\sigma(W(L)), \rho$, such that $0 \leq \rho \leq \hat{\mu}, \rho$ is $\delta W(L)$-regular, and $\rho^{*}(H)=\rho(\operatorname{IR}(L))=\hat{\mu}^{*}(H)$.

Саse 2: There exists a countably additive measure on $\sigma(t W(L)), \rho$, such that $0 \leq \rho \leq \tilde{\mu}, \rho$ is $\operatorname{tW}(L)$-regular, and $\rho^{*}(H)=\rho(\operatorname{IR}(L))=\tilde{\mu}^{*}(H)$.

(See [5], Lemma 4.1.)

THEOREM 3.3. The following statements are equivalent:

1. $L$ is measure replete.

2. For every element of $\operatorname{MR}(\sigma, L)-\{0\}, \mu, S(\mu) \neq \emptyset$.

Proof. a) Assume 1, and show 2. Consider any element of $\operatorname{MR}(\sigma, L)-\{0\}, \mu$. Since $L$ is measure replete, $\operatorname{MR}(\sigma, L) \subset \operatorname{MR}(\tau, L)$. Consequently $\mu \in \operatorname{MR}(\tau, L)-\{0\}$. Hence $S(\mu) \neq \emptyset$.

B) Conversely, assume 2 , and show 1 . Note to show $L$ is measure replete, it suffices to show $\operatorname{MR}(\sigma, L) \subset \operatorname{MR}(\tau, L)$. Consider any element of $\operatorname{MR}(\sigma, L)-\{0\}, \mu$. Assume $\mu \notin \mathbb{M R}(\tau, L)$. Then, by Theorem 1.1, part 2, there exists an element of $t W(L), K$, such that $K \subset \operatorname{IR}(L)-X$ and $\tilde{\prod}(K) \neq 0$. Consider any such $K$. Then, by Lemma 3.2, Case 2, there exists a countably additive measure on $\sigma(t W(L)), \rho$, such that $0 \leq \rho \leq \tilde{\mu}$, $\rho$ is $\mathrm{tW}(L)$-regular, and $\rho^{*}(K)=\rho(\operatorname{IR}(L))=\tilde{\mu}^{*}(K)$. Consider any such $\rho$. Now, consider $\left.\rho\right|_{A(W(L))}$ and the element of $M(L), \nu$, which is such that $\left.\rho\right|_{A(W(L))}=\hat{v}$. Note $\left.\rho\right|_{A(W(L))} \in \operatorname{MR}(W(L))$. Consequent1y $v \in \operatorname{MR}(L)$.

Show $\nu \in \mathbb{M R}(\sigma, L)$. Use Theorem 1.1, part 1. Consider any sequence in $L,\left\langle L_{1}>\right.$, such that $\left\langle L_{i}>\right.$ is decreasing and $\underset{i}{n} W\left(L_{i}\right) \subset \operatorname{IR}(L)-X$, and show $\hat{v}\left(\underset{i}{n W}\left(L_{i}\right)\right)=0$. Note $\prod_{i} W\left(L_{i}\right) \in \sigma(W(L))$ and, since $\left.\rho\right|_{\sigma(W(L))}=\hat{v}$ (by Uniqueness of Extension), $\hat{v}\left(n_{i} W\left(L_{i}\right)\right)=$ $\rho\left(\operatorname{NW}_{i}\left(L_{1}\right)\right)$. Since $\rho \leq \tilde{\mu},\left.\rho\right|_{\sigma(W(L))} \leq\left.\tilde{\mu}\right|_{\sigma(W(L))}$. Further, note $\left.\tilde{\mu}\right|_{\sigma(W(L))}=\hat{\mu}$, by Uniqueness of Extension. Consequent1y $\left.\rho\right|_{\sigma(W(L))} \leq \hat{\mu}$. Consequently $\hat{v}\left(\cap_{i}\left(L_{i}\right)\right)=$ 
$\rho\left(\mathrm{nW}_{i}\left(\mathrm{~L}_{\mathbf{i}}\right)\right) \leq \hat{\mu}\left(\mathrm{nW}_{\mathbf{i}}\left(\mathrm{L}_{\mathbf{i}}\right)\right)$. Since $\mu \in \mathrm{MR}(\sigma, L)$, by Theorem 1.1, part $1, \hat{\mu}\left(\mathrm{nW}_{\mathbf{i}}\left(\mathrm{L}_{\mathbf{i}}\right)\right)=0$. Consequently $\hat{v}\left(\mathrm{nW}_{i}\left(\mathrm{~L}_{i}\right)\right)=0$. Then, by Theorem 1.1, part $1, v \in \mathbb{M}(\sigma, L)$. Moreover, since $v(X)=\hat{v}(\operatorname{IR}(L))=\rho(\operatorname{IR}(L))=\tilde{\mu}^{*}(K)=\tilde{\mu}(K) \neq 0, v \neq 0$. Then, by the assumption, $\mathrm{s}(\nu) \neq \emptyset$.

Further, note $S(v)=S(\tilde{v}) \cap X$. Also, since $\left.\rho\right|_{A(W(L))}=\hat{v}$ and $W(L)$ separates $t W(L),\left.\quad \rho\right|_{A(t W(L))}=\sigma$. Consequently $S(v)=S(\rho) \cap x$. Moreover, since $\rho^{*}(K)=$ $\rho(\operatorname{IR}(L))$ and $\rho^{*}(K)=\rho(K), S(\rho) \subset K$. Hence, since $K \subset \operatorname{IR}(L)-X, S(\rho) \cap X=\emptyset$. Consequently $S(\nu)=\emptyset$. Thus a contradiction has been reached. Consequently $\mu \in \operatorname{MR}(\tau, L)$. Hence $\operatorname{MR}(\sigma, L) \subset \operatorname{MR}(\tau, L)$. Consequently $L$ is measure replete.

Remark. This theorem generalizes [8], Theorem 2.2, where it is assumed that $L$ is $\delta$.

Examples. (1). Consider any topological space $X$ such that $X$ is $T_{3 \frac{1}{2}}$ and let $L=Z$. Then, by Theorem 3.3, (or by [8], Theorem 2.2), $X$ is measure compact iff for every element of $\operatorname{MR}(\sigma, Z)-\{0\} \mu, \quad S(\mu) \neq \emptyset$.

(2). Consider any topological space $X$ such that $X$ is $T_{1}$ and let $L=F$. Then, by Theorem 3.3, (or by [8], Theorem 2.2), $x$ is Borel measure compact iff for every element of $\operatorname{MR}(\sigma, F)-\{0\}, \mu, S(\mu) \neq \emptyset$.

(3). Consider any topological space $X$ such that $X$ is $T_{1}$ and 0 -dinensional and let $L=C$. Then, by Theorem 3.3, $X$ is clopen measure replete iff for every element of $\operatorname{MR}(\sigma, C)-\{0\}, \mu, S(\mu) \neq \emptyset$.

$\gamma)$ Other properties of support.

Theorem 3.4. The following statements are equivalent:

1. $L$ is regular.

2. For every two elements of $M(L), \mu, \nu$, if $\mu \leq \nu$ on $L$ and $\mu(X)=\nu(X)$, then $S(\mu)=S(\nu)$.

Proof. $\alpha)$ Assume 1, and show 2. Consider any two elements of $M(L), \mu, \nu$, such that $\mu \leq \nu$ on $L$ and $\mu(X)=\nu(X)$. Since $\mu \leq \nu$ on $L$ and $\mu(X)=\nu(X), S(\nu) c$ $S(\mu)$. Assume $S(\mu) \neq S(\nu)$. Then there exists an element of $S(\mu), x$, such that $x \notin S(\nu)$. Consider any such $x$. Then, since $S(\nu)=n\{L \in L / \nu(L)=\nu(x)\}$, there exists an element of $L, L$, such that $\nu(L)=\nu(X)$ and $x \notin L$. Consider any such $L$. Then, since $L$ is regular, there exist two elements of $L, A, B$, such that $x \in A^{\prime}$ and $L \subset B^{\prime}$ and $A^{\prime} \cap B^{\prime}=\emptyset$. Consider any such $A, B$. Then $L \subset B^{\prime} \subset A$. Consequently $\mu(X)=\nu(X)=\nu(L) \leq \nu\left(B^{\prime}\right) \leq \mu\left(B^{\prime}\right) \leq \mu(A) \leq \mu(X)$. Consequent1y $A \in L$ and $\mu(A)=\mu(X)$. Hence, since $x \in S(\mu), \quad x \in A$. Thus a contradiction has been reached. Consequently $S(\mu)=S(\nu)$.

B) Conversely, assume 2, and show 1. Note, by the assumption, for every two elements of $I(L), \mu, \nu$, if $\mu \leq \nu$ on $L$, then $S(\mu)=S(\nu)$. Then, $L$ is regular. (Proof known, see, e.g., [6].)

Remark 1. The condition " $L$ is separating and disjunctive" was not needed in the proof.

Remark 2. This theorem generalizes a known result on $0-1$ measures.

Observation 1. Assume $L$ is regular. Consider any element of $M(L), \mu$. Then there exists an element of $\operatorname{MR}(L), \nu$, such that $\mu \leq \nu$ on $L$ and $\mu(X)=\nu(X)$. (See 
e.g.,[10].) Consider any such $\nu$. Then, since $L$ is regular, by Theorem $3.4, S(\mu)=$ $\mathrm{s}(\nu)$.

The significance of this observation is this: Given that $L$ is regular, in checking facts about the support of an element of $M(L), \mu$, it is permissible to assume $\mu \in \operatorname{MR}(L)$.

Observation 2. Assume $L$ is regular and countably compact. Consider any element of $M(L), \mu$. Then, consider any element of $\operatorname{MR}(L), \nu$, such that $\mu \leq \nu$ on $L$ and $\mu(X)=\nu(X)$. Since $L$ is countably compact, $\operatorname{MR}(L)=\operatorname{MR}(\sigma, L)$. Consequently $\nu \in \mathbb{R}(\sigma, L)$. Moreover, since $L$ is regular, $S(\mu)=S(\nu)$.

The significance of this observation is this: Given that $L$ is regular and countably compact, in checking facts about the support of an element of $M\left(\sigma^{*}, L\right), \mu, 1 t$ is permissible to assume $\mu \in \operatorname{MR}(\sigma, L)$.

Example. Consider any topological space $X$ such that $X$ is regular and countably compact and let $L=F$. Then, by observation 2 , for every element of $M\left(\sigma^{*}, F\right)$, $\mu$, there exists an element of $\operatorname{MR}(\sigma, F)$, that is, a Borel measure, $\nu$, such that $\mu \leq \nu$ on $F$ and $\mu(X)=\nu(X)$ and $S(\mu)=S(\nu)$.

Observation 3. Assume $L$ is regular, normal, and countably paracompact. Consider any element of $M\left(\sigma^{*}, L\right), \mu$. Then, since $L$ is normal and countably paracompact, there exists an element of $\operatorname{MR}(\sigma, L), \nu$, such that $\mu \leq \nu$ on $L$ and $\mu(X)=$ $v(\mathrm{X})$. (See [8], Theorem 4.1) Consider any such $\nu$. Then, since $L$ is regular, by Theorem 3.4, $S(\mu)=S(\nu)$.

The significance of this observation is this: Given that $L$ is regular, normal, and countably paracompact, in checking facts about the support of an element of $M\left(\sigma^{*}, L\right), \mu$, it is permissible to assume $\mu \in \operatorname{MR}(\sigma, L)$.

Example. Consider any topological space $X$ such that $X$ is $T_{3 \frac{1}{2}}$ and 1 et $L=Z$. Then, by Observation 3 , for every element of $M\left(\sigma^{*}, Z\right), \mu$, there exists an element of $\operatorname{MR}(\sigma, Z)$, that is, a Baire measure, $\nu$, such that $\mu \leq \nu$ on $Z$ and $\mu(X)=\nu(X)$ and $S(\mu)=S(\nu)$.

Observation 4. Consider any topological space $X$ such that $X$ is $T_{3 \frac{1}{2}}$ and pseudocompact and let $L=F$. Now, consider any element of $M(F)$, $\rho$. Note $\rho \in M(0)$. Consider any element of $M R(O), \nu$, such that $\rho \leq \nu$ on $O$ and $\rho(X)=\nu(X)$. Then $\nu \leq \rho$ on $F$.

Next, show $\nu \in M\left(\sigma^{*}, F\right)$. Assume $\nu \notin M\left(\sigma^{*}, F\right)$. Then there exists a sequence in $F$, $\left\langle F_{n}\right\rangle$, such that $\left\langle F_{n}>\right.$ is decreasing and $\underset{n}{\lim } F_{n}=\emptyset$, but $\underset{n}{\lim } \nu\left(F_{n}\right) \neq 0$. Consider any such $\left\langle F_{n}>\right.$. Since $\left\langle F_{n}\right\rangle$ is decreasing and $\lim _{n} F_{n}=\emptyset$, $<F_{n}^{\prime}>$ is increasing and $\underset{n}{U} F_{n}^{\prime}=X$. Since $X$ is pseudocompact, there exists a value of $n$, $n_{0}$, such that $\underset{k=1}{\mathrm{U}} \bar{F}_{k}^{\prime}=\mathrm{X}$. Consider any such $\mathrm{n}_{0}$. Since $\left\langle\mathrm{F}_{\mathrm{n}}^{\prime}>\right.$ is increasing, $\left\langle\overline{\mathrm{F}}_{\mathrm{n}}^{\prime}>\right.$ is increasing. Consequently, $\overline{\mathrm{F}}_{\mathrm{n}_{0}}^{\prime}=\mathrm{x}$ and for every $\mathrm{n}$, if $\mathrm{n} \geq \mathrm{n}_{0}$, then $\overline{\mathrm{F}}_{\mathrm{n}}^{\prime}=\mathrm{x}$. Now, note since $\lim _{n} \nu\left(F_{n}\right) \neq 0, \lim \nu\left(F_{n}\right)>0$. Set $\lim _{n} \nu\left(F_{n}\right)=\varepsilon$. Then for every $n$, since $\nu \in \operatorname{MR}(O)$, there exists an element of $O, G_{n}$, such that $G_{n} \subset F_{n}$ and $\nu\left(G_{n}\right)>\varepsilon$; consider any such $G_{n}$; then $\bar{G}_{n} \subset F_{n}$; hence $\bar{G}_{n}^{\prime} \supset F_{n}^{\prime}$; consequently $G_{n}^{\prime} \supset \bar{r}_{n}^{\prime} \supset F_{n}^{\prime}$; hence 
$G_{n}^{\prime} \supset \bar{F}_{n}^{\prime}$. Consequently for every $n$, if $n \geq n_{0}$, then $G_{n}^{\prime} \supset \bar{F}_{n}^{\prime}=x$. Hence for every $n$, if $\mathrm{n} \geq \mathrm{n}_{0}$, then $\mathrm{G}_{\mathrm{n}}=\emptyset$. Thus a contradiction has been reached. Consequently $\nu \epsilon$ $M\left(\sigma^{*}, L\right)$.

Finally, note since $X$ is completely regular, it is regular; equivalently, $F$ is regular. Then, by Theorem 3.4, $S(\rho)=S(\nu)$.

The significance of this observation is this: Given that $\mathrm{X}$ is $\mathrm{T}_{3 \frac{1}{2}}$ and pseudocompact, in checking facts about the support of an element of $M(F)$, $\rho$, it is permissible to assume $\rho \in M\left(\sigma^{*}, F\right)$.

The purpose of the following discussion is to illustrate the importance of considering $\sigma$-smoothness with respect to lattices.

Lemma 3.3. If $L$ is complement generated, then $M\left(\sigma^{*}, L\right) \subset M R(L)$.

Proof. Assume $L$ is complement generated. Consider any element of $M\left(\sigma^{*}, L^{\prime}\right), \mu$ Note to show $\mu \in \operatorname{MR}(L)$, it suffices to show for every element of $L, L, \mu(L)=$ $\inf \left\{\mu\left(\tilde{L}^{\prime}\right) / \tilde{L} \in L\right.$ and $\left.\tilde{L}^{\prime} \supset L\right\}$. Consider the function $\rho$ which is such that $D_{\rho}=L$ and for every element of $L, L, \rho(L)=\inf \left\{\mu\left(\tilde{L}^{\prime}\right) / \tilde{L} \in L\right.$ and $\left.\tilde{L}^{\prime} \supset L\right\}$. Show for every element of $L, L, \rho(L)=\mu(L)$. Consider any element of $L$, $L$. Note $\mu(L) \leq \rho(L)$. Now, show $\rho(L) \leq \mu(L)$. Since $L \in L$ and $L$ is complement generated, there exists a sequence in $L$, $\left\langle L_{n}>\right.$, such that $L=n_{n} L_{n}^{\prime}$. Consider any such $<L_{n}>$. Without loss of generality, assume $\left\langle L_{n}^{\prime}>\right.$ is decreasing. Note for every $n, \mu\left(L_{n}^{\prime}\right)=\mu\left(L_{n}^{\prime} \cap L\right)+\mu\left(L_{n}^{\prime} \cap L^{\prime}\right)$. Since $L={ }_{n} L_{n}^{\prime}, L_{n}^{\prime} \cap L=L$. Next, consider $\left\langle L_{n}^{\prime} \cap L^{\prime}\right\rangle$. Note $\left\langle L_{n}^{\prime} \cap L^{\prime}>\right.$ is in $L^{\prime}$ and since $\left\langle\mathrm{L}_{\mathrm{n}}^{\prime}>\right.$ is decreasing, $\left\langle\mathrm{L}_{\mathrm{n}}^{\prime} \cap \mathrm{L}^{\prime}>\right.$ is decreasing and since $\mathrm{L}^{n} \mathrm{n}_{\mathrm{n}}^{\prime}$, $\lim _{n}\left(L_{n}^{\prime} \cap L^{\prime}\right)=\bigcap_{n}\left(L_{n}^{\prime} \cap L^{\prime}\right)=\left(\bigcap_{n}^{\prime} L_{n}^{\prime}\right) \cap L^{\prime}=\emptyset$. Then, since $\mu \in M\left(\sigma^{*}, L^{\prime}\right), \lim _{n} \mu\left(L_{n}^{\prime} \cap L^{\prime}\right)=$ 0. Consequently $\lim _{n} \mu\left(L_{n}^{\prime}\right)=\lim _{n} \mu\left(L_{n}^{\prime} \cap L\right)+\lim _{n} \mu\left(L_{n}^{\prime} \cap L^{\prime}\right)=\mu(L)+0=\mu(L)$. Consequently $\rho(L) \leq \mu(L)$. Then $\rho(L)=\mu(L)$. Thus $\mu(L)=\inf \left\{\mu\left(\tilde{L}^{\prime}\right) / \tilde{L} \in L\right.$ and $\left.\tilde{L}^{\prime} \supset L\right\}$. Consequently $\mu \in \operatorname{MR}(L)$. Hence $M\left(\sigma^{*}, L^{\prime}\right) \subset \operatorname{MR}(L)$.

Lemma 3.4. If $L$ is countably paracompact, then $M\left(\sigma^{*}, L^{\prime}\right) \subset M\left(\sigma^{*}, L\right)$.

Proof. Assume $L$ is countably paracompact. Consider any element of $M\left(\sigma^{*}, L^{\prime}\right), \mu^{\prime}$ To show $\mu \in M\left(\sigma^{*}, L\right)$, consider any sequence in $L,\left\langle L_{n}>\right.$, such that $<L_{n}>$ is decreasing and $\lim _{n} L_{n}=\emptyset$, and show $\lim _{n} \mu\left(L_{n}\right)=0$. Since $\left\langle L_{n}>\right.$ is in $L$ and $\left\langle L_{n}>\right.$ is decreasing and $\lim _{n} L_{n}=\emptyset$ and $L$ is countably paracompact, there exists a sequence in $L, \quad\left\langle\tilde{L}_{n}>\right.$, such that for every $n, L_{n} \subset \tilde{L}_{n}^{\prime}$ and $\left\langle\tilde{L}_{n}^{\prime}\right\rangle$ is decreasing and $\lim _{n} \tilde{L}_{n}^{\prime}=$ $\emptyset$. Consider any such $\left\langle\tilde{L}_{n}>\right.$. Then, since $\mu \in M\left(\sigma^{*}, L^{\prime}\right), \lim _{n} \mu\left(\tilde{L}_{n}^{\prime}\right)=0$. Consequently $\lim _{\mathrm{n}} \mu\left(\mathrm{L}_{\mathrm{n}}\right)=0$. Thus $\mu \in M\left(\sigma^{*}, L\right)$. Hence $M\left(\sigma^{*}, L^{\prime}\right) \subset M\left(\sigma^{*}, L\right)$.

THEOREM 3.5. If $L$ is complement generated, then $M\left(\sigma^{*}, L^{\prime}\right)=\operatorname{MR}(\sigma, L)$.

Proof. Assume $L$ is complement generated. Note in general, $\operatorname{MR}(\sigma, L) \subset M\left(\sigma^{*}, L^{\prime}\right)$. Now, show $M\left(\sigma^{*}, L^{\prime}\right) \subset \operatorname{MR}(\sigma, L)$.

a) Since $L$ is complement generated, by Lemma $3.3, M\left(\sigma^{*}, L^{\prime}\right) \subset \operatorname{MR}(L)$.

B) Since $L$ is complement generated, $L$ is countably paracompact. Hence, by Lemma 3.4, $M\left(\sigma^{*}, L^{\prime}\right) \subset M\left(\sigma^{*}, L\right)$. 


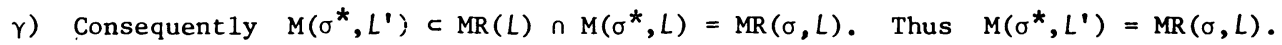
Observation. Note $M(\sigma, L) \subset M\left(\sigma^{*}, L^{\prime}\right)=M R(\sigma, L) \subset M(\sigma, L)$. Hence $M(\sigma, L)=M R(\sigma, L)$. This result generalizes the following well-known result: Consider any topological space $X$ such that $X$ is $T_{3 \frac{1}{2}}$ and let $L=Z$. Then $M(\sigma, Z)=M R(\sigma, Z)$; expressed otherwise: every Baire measure is regular.

Remark. Note the condition " $L$ is separating and disjunctive" was not needed in the proof.

Finally, we will consider the question of when the support of a measure is Lindelöf.

Proposition 3.1. ( $\alpha$ ) If $L$ is Lindelof (compact), then for every element of $M(L)$, $\mu, S(\mu)$ is Lindelöf (conpact).

B) If $L$ is $\delta$, then for every element of $\operatorname{MR}(\tau, L), \mu, \mu^{*}(S(\mu))=\mu(X)$.

$\gamma)$ For every element of $\operatorname{MR}(\sigma, L), \mu$, if $S(\mu)$ is Lindelof, then for every element of $\operatorname{MR}(t L), \nu$, if $\nu \mid A(L)=\mu$, then $\nu(S(\mu))=\mu^{*}(S(\mu))$.

(Proof omitted.)

Remark. The condition " $L$ is separating and disjunctive" was not needed in the proof.

Definition. $L$ is paracompact iff $L$ is regular and for every subset of $L, \quad\left\{A_{\alpha} ; \alpha \in \Lambda\right\}$, if $\cup\left\{A_{\alpha}^{\prime} ; \alpha \in \Lambda\right\}=X$, then there exists a subset of $L,\left\{B_{\alpha}\right.$; $\alpha \in \Lambda\}$, such that for every $\alpha, B_{\alpha}^{\prime} \subset A_{\alpha}^{\prime}$ and $U\left\{B_{\alpha}^{\prime} ; \alpha \in \Lambda\right\}=X$ and for every element of $\mathrm{x}, \mathrm{x}$, there exists an element of $L, L$, such that $\mathrm{x} \in \mathrm{L}^{\prime}$ and $\left\{\alpha \in \Lambda / L^{\prime} \cap \mathrm{B}_{\alpha}^{\prime} \neq \emptyset\right\}$ is finite.

THEOREM 3.6. If $t L$ is paracompact, then for every element of $M R(\tau, L), \mu, S(\mu)$ is Lindelof.

Proof. Assume $t L$ is paracompact. Consider any element of $\operatorname{MR}(\tau, L)-\{0\}, \mu$. Then $S(\mu) \neq \emptyset$. Consider any subset of $(t L)^{\prime},\left\{0_{\alpha} ; \alpha \in A\right\}$, such that $S(\mu) \subset \cup\left\{0_{\alpha}\right.$; $\alpha \in \mathrm{A}$ \}。 Note $\mathrm{S}(\mu) \in \mathrm{tL}$. Hence, since $t \mathrm{~L}$ is paracompact, $\mathrm{S}(\mu) \cap t L$ is paracompact. Then there exists an open refinement of $\left\{0_{\alpha} ; \alpha \in A\right\}, G$, such that $S(\mu) \subset \cup G$ and there exists a sequence of subsets of $G,\left\langle G_{n}>\right.$, such that $G=U G_{n}$ and for every $n$, $G_{\mathrm{n}}$ is discrete. Consider any such $G$ and any such $<G_{\mathrm{n}}>$. Note to show there exists a subset of $A, A^{*}$, such that $S(\mu) \subset U\left\{0_{\alpha} ; \alpha \in A^{*}\right\}$ and $A^{*}$ is countable, it suffices to show that for every $n, G_{n}$ is countable. To do this, proceed as follows:

Since $L$ is separating and disjunctive, and $\mu \in \operatorname{MR}(\tau, L)$, by [5], Theorem 2.5, there exists an element of $\operatorname{MR}(\tau, t L), v$, such that $\left.\nu\right|_{A(L)}=\mu$ and $v$ is unique.

observation. For every element of $L, L$, if $S(\mu) \cap L^{\prime} \neq \emptyset$, then $\nu\left(S(\mu) \cap L^{\prime}\right)>0$. (Proof omitted.) Since $S(\mu) \subset U G$ and $S(\mu) \neq \emptyset$, without loss of generality assume for every element of $G, 0, S(\mu) \cap 0 \neq \emptyset$.

Now, consider any $n$. Since $G_{n}$ is discrete, $G_{n}$ is disjoint; hence $\{S(\mu) \cap 0$; $\left.0 \in G_{n}\right\}$ is disjoint. Therefore to show $G_{n}$ is countable, it suffices to show $\left\{S(\mu) \cap 0 ; 0 \in G_{n}\right\}$ is countable; consider any element of $G_{n}, 0$; then, by assumption, $S(\mu) \cap 0 \neq \emptyset$; consider any element of $S(\mu) \cap 0, x$; then, there exists an element of $L$, 
$\tilde{\mathrm{L}}$, such that $\mathrm{x} \in \tilde{\mathrm{L}}^{\prime} \subset 0$; consider any such $\tilde{\mathrm{L}}$; then, by the observation, $\nu\left(\mathrm{S}(\mu) \mathrm{n} \tilde{L}^{\prime}\right)$

$>0$; consequently $\nu(S(\mu) \cap 0)>0$. Therefore $\left\{S(\mu) \cap 0 ; 0 \in G_{n}\right\}$ is countable;

(proof omitted;) consequently $G_{n}$ is countable.

Whence $S(\mu)$ is Lindelöf.

Examples. (1). Consider any topological space $\mathrm{X}$ such that $\mathrm{X}$ is $\mathrm{T}_{1}$ and paracompact. (Note $\mathrm{X}$ is $\mathrm{T}_{3 \frac{1}{2}}$ ). Let $L=Z$. Then by Theorem 3.6 , for every element of $\operatorname{MR}(\tau, Z), \mu, S(\mu)$ is Linde18f. (This result is known.)

(2). Consider any topological space $X$ such that $X$ is $T_{1}$ and paracompact and $L=F$. Then, by Theorem 3.6, for every element of $\operatorname{MR}(\tau, F), \mu, S(\mu)$ is Lindelif. (3). Consider any topological space $X$ such that $X$ is $T_{1}$ and 0 -dimensional and paracompact. Let $L=C$. Then, by Theorem 3.6, for every element of $M R(\tau, C) ; \mu$, $S(\mu)$ is Lindelof.

ACKNOWLEDGEMENT: The second author wishes to express his appreciation to Long Island University for partial support of the present work through a grant of released time from teaching duties.

\section{REFERENCES}

1. ALEXANDROFF, A. D., Additive Set Functions in Abstract Spaces, Mat. Sb. (N. S.) 8 $\underline{50}$ (1940) 307-348.

2. Additive Set Functions in Abstract Spaces, Mat. Sb. (N.S.) 9 51(1941) 563-628.

3. BACHMAN, G., and HSU, P., Extensions of Lattice Continuous Maps to Generalized Wallman Spaces, Atti Accad. Naz. Lincei Rend. C1. Sc. Fis. Mat. Natur., 62 (1977) 107-114.

4. BACHMAN, G. and SULTAN, A., On Regular Extensions of Measures, Pacific J. Math, 86 (1980) 389-395.

5. BACHMAN, G. and STRATIGOS, P., Criteria for $\sigma$-smoothness, $\tau$-smoothness, and tightness of Lattice Regular Measures, with Applications, Can. J. Math., 33 (1981) 1498-1525.

6. FROLIK, Z., Prime Filters with the C.I.P., Comm. Math. Univ. Carolinae, 13 (1972) 553-575.

7. NOEBELING, G., Grundlagen der analytischen topologie, Springer-Verlag, Berlin 1954.

8. SZETo, M. Measure Repleteness and Mapping Preservations, J. Indian Math. Soc. 43 (1979) 35-52.

9. "On Maxima1 Measures with:!Respect to a Lattice," in Measure Theory and its Applications, proceedings of the 1980 conference, (edited by G. Goldin and P.F. Wheeler) Northern Illinois University Press, DeKalb, I11, (1980) 277-282.

10. WALEMAN,H., Lattices and Topological Spaces, Ann. Math., 39(1938) 112-126. 


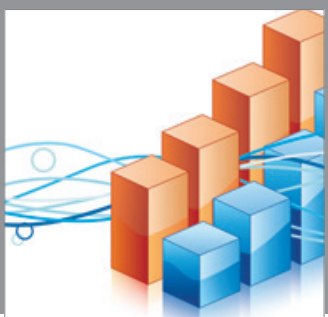

Advances in

Operations Research

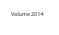

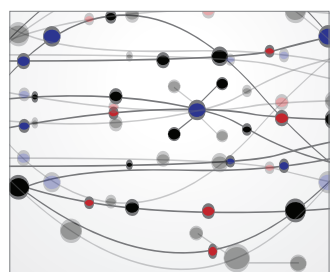

\section{The Scientific} World Journal
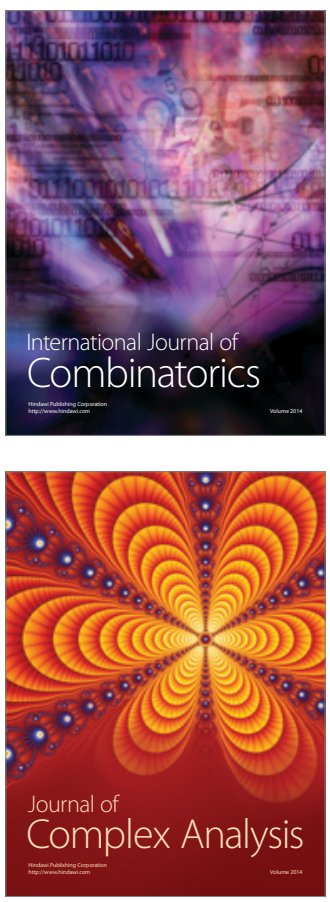

International Journal of

Mathematics and

Mathematical

Sciences
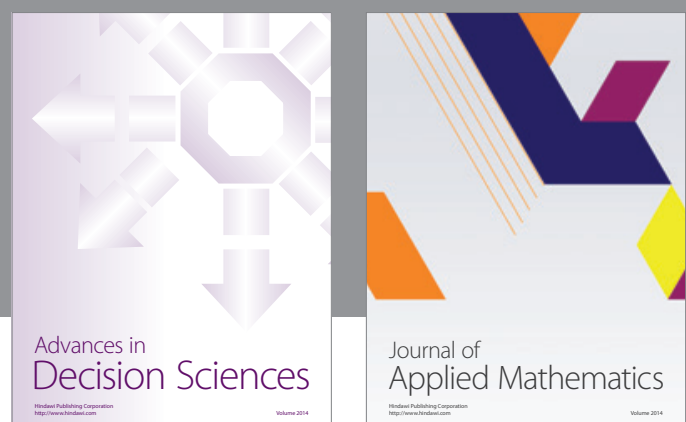

Journal of

Applied Mathematics
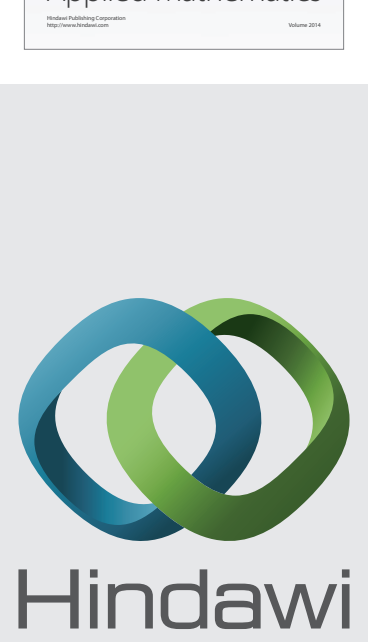

Submit your manuscripts at http://www.hindawi.com
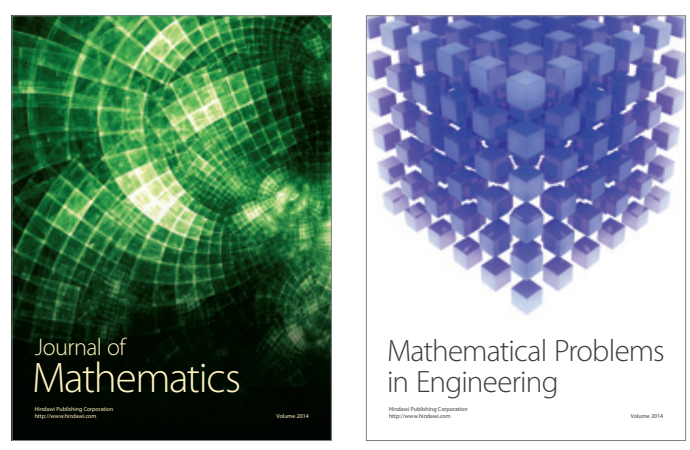

Mathematical Problems in Engineering
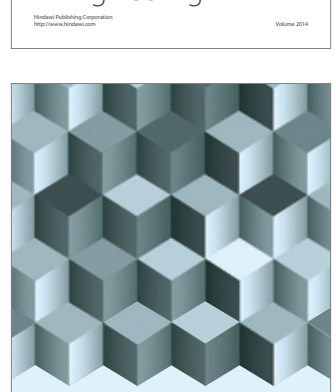

Journal of

Function Spaces
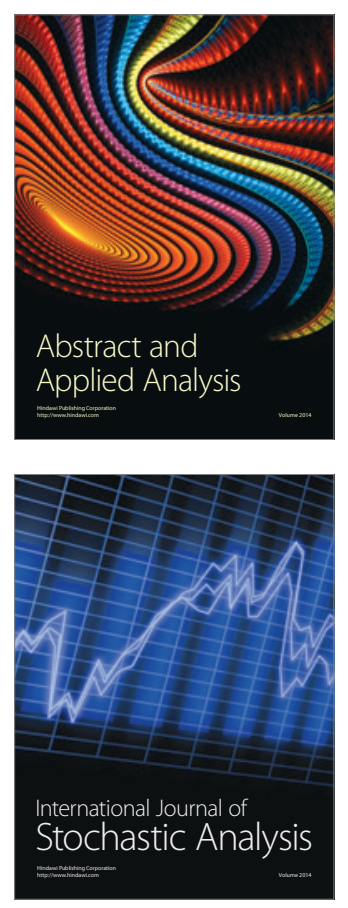

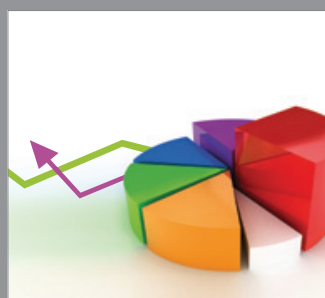

ournal of

Probability and Statistics

Promensencen
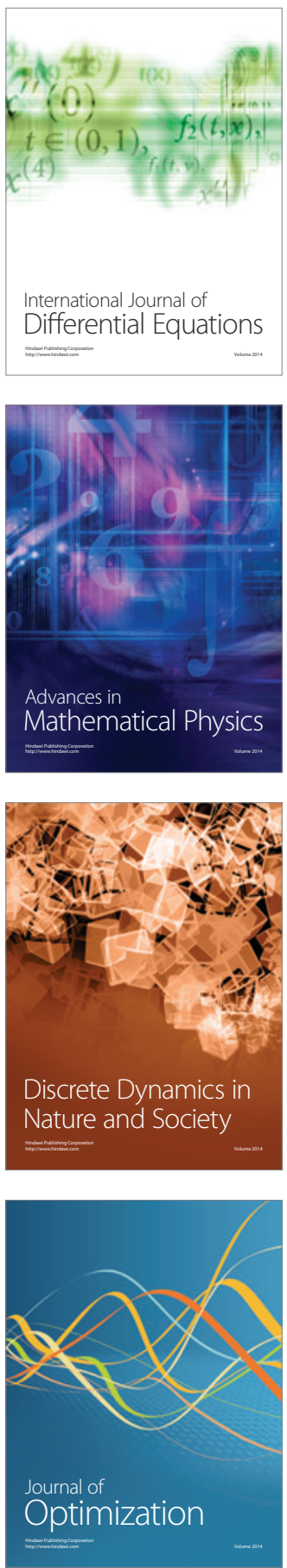